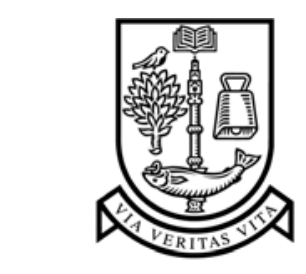

UNIVERSITY

of

GLASGOW

Ramloll, R. and Brewster, S.A. (2002) A generic approach for augmenting tactile diagrams with spatial non-speech sounds. In, Conference on Human Factors in Computing Systems, 20-25 April 2002, pages pp. 770-771, Minneapolis, Minessota, USA.

http://eprints.gla.ac.uk/3227/ 


\title{
A Generic Approach for Augmenting Tactile Diagrams with Spatial Non-Speech Sounds
}

\author{
Rameshsharma Ramloll and Stephen Brewster \\ Glasgow Interactive Systems Group, Department of Computing Science, University of \\ Glasgow, UK \\ +44 (0)141 330 4966, \{ramesh, stephen\}@dcs.gla.ac.uk, www.dcs.gla.ac.uk/ stephen
}

\begin{abstract}
Blind or visually impaired users typically access diagrams in the tactile medium. This paper describes TouchMelody, a system designed for augmenting such existing diagrams with 3D spatial auditory information to increase their use-fulness, information content and reduce tactile clutter. The motivation for this system, an overview of its development and early experiences are presented. The two major technologies used are the Polhemus FASTRAK and the LakeDSP CP4 to facilitate the creation of a directly ma-nipulatable dynamic 3D spatial soundscape.
\end{abstract}

\section{Keywords}

Tactile diagrams, sound enhancement, spatial sound

\section{INTRODUCTION}

A study of browsing practices of tactile diagrams (e.g. graphs, tables and simple geometric shapes) by a sample of blind and visually impaired individuals was carried out at the Glasgow and West of Scotland Society for the Blind in the UK. It revealed that browsing strategies were very user specific and that education played a major role in the way participants approached such diagrams. Most participants found the task of interpreting such diagrams difficult. One common browsing strategy was to use the nondominant hand to situate a point of reference on the tactile diagram and the dominant hand to explore the scene relative to this. Our application, TouchMelody, was designed around this kind of two-handed interaction.

Tactile diagrams are static; the information they contain cannot be changed. The basic design of such diagrams (us-ing special paper with raised lines) has not changed over recent years and there is much information stored in this format in libraries and used for teaching. Being able to en-hance this existing material with dynamic feedback will improve its usefulness and usability. Our approach overlays the diagrams with non-speech audito information, based on the idea of Soundgraphs [3, 4]. This is generic because we can overlay any existing diagrams with audio information.

A basic guideline [1, 2] for the construction of tactile dia-grams is to avoid "clutter" and simplify content. "Clutter" occurs when different symbols and lines are so close and/or so similar that they become hard to distinguish. Spacing has traditionally been the key to avoiding clutter. Our system allows the transfer of some information from the tactile medium into the auditory to help solve this problem. For example, grid lines needed to add meaning to tactile graphs are known to increase tactile "clutter". Readers often find it difficult to distinguish between the grid lines and the actual graphs. TouchMelody can eliminate the need for tactile grid lines by presenting them in sound.

\section{THE STRUCTURE OF TOUCHMELODY}

The main components of our system are a Polhemus FAS-TRAK (www.polhemus.com) motion tracker and a LakeDSP CP4 (www.lakedsp.com) 3D audio soundcard together with its Multiscape application to create the 3D soundscape. There are two sound objects: an observer (O in Figure 1, normally the non-dominant hand providing the anchor point) and a streamed sound source (S in Figure 1, the dominant hand used for exploration). The positions of $\mathrm{O}$ and $\mathrm{S}$ in the virtual soundscape are managed by Multis-cape which continuously receives the current positions of the FASTRAK sensors mounted on the index fingers (Fig-ure 1 , left inset) with their tips free to allow normal reading of the diagram. The user can then hear the location of $\mathrm{S}$ relative to $\mathrm{O}$ as he/she moves in real time; if $\mathrm{S}$ is to the right of $\mathrm{O}$ the sound heard will appear to becoming from the right and vice versa. Figure 2 shows a general view of our hardware setup. 


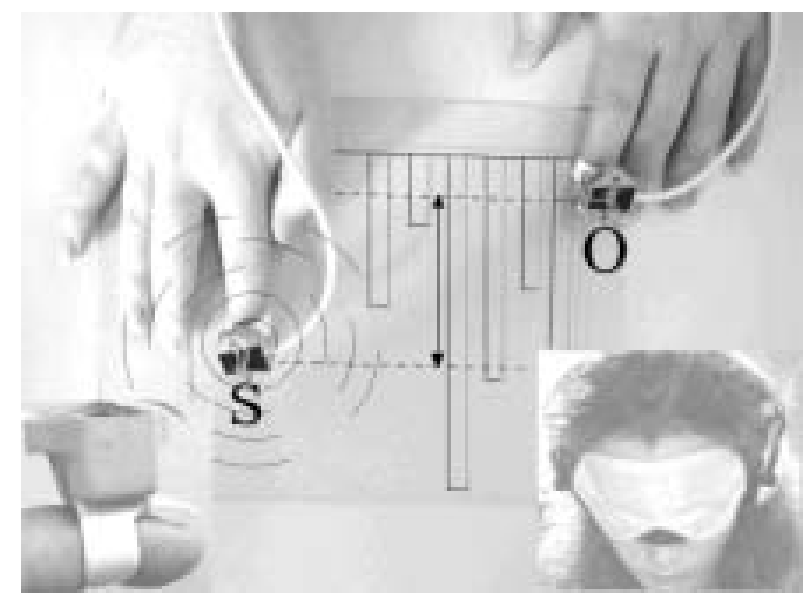

Figure 1: Using TouchMelody to explore a raised bar chart.

$\mathrm{S}$ produces a stream of piano notes $20 \mathrm{~ms}$ apart whenever both fingers touch the diagram; the visually impaired user is able to listen to and locate the position of the source (S) through the headphones (Figure 1, right inset). The pitch of the stream at $\mathrm{S}$ also changes with the vertical distance be-tween $\mathrm{S}$ and $\mathrm{O}$ (as illustrated by the arrow). A user moving $\mathrm{S}$ relative to $\mathrm{O}$ will hear changes in the pitch and also get some idea of the direction and proximity of the source. The pitches provide similar information to that of tactile grid lines, but without the clutter. The pitches produced can be used to reinforce or disambiguate tactile comparisons of the raised bars in the graph. In this way, our system can be used in conjunction with any existing raised diagrams commonly used by blind people.

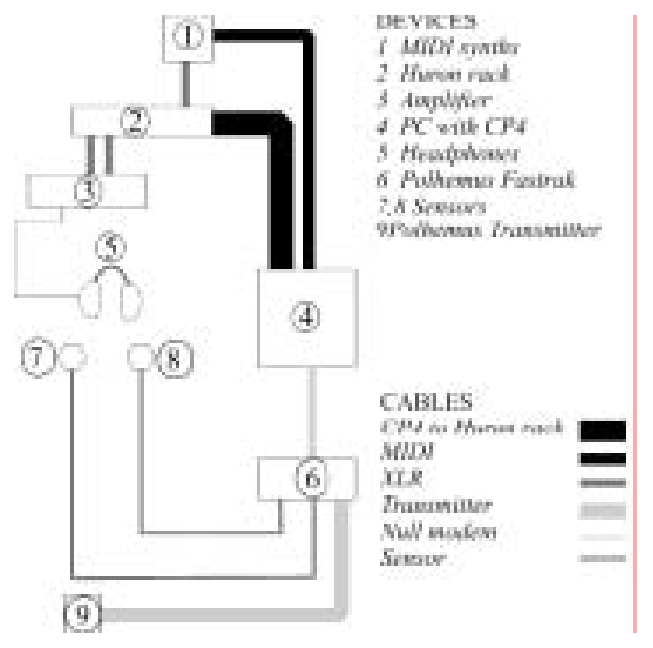

Figure 2: TouchMelody's hardware setup.

\section{SOUND MAPPINGS}

The pitch of the virtual streamed source $S$ (i.e. the pitch of each piano note in the sequence) depends on the vertical relationship between $\mathrm{O}$ and $\mathrm{S}$ (Figure 3, $\mathrm{T}$ is the FAS-TRACK transmitter). This is similar way to a standard Soundgraph $[3,4]$ where the value on the y axis of the graph is mapped to pitch and the $\mathrm{x}$ axis to time. The follow-ing empirically derived equation gives the pitch of the streamed source (as a MIDI note number):

$$
\text { MIDIMLmber }=40+(\text { displacement } / \text { sweep }) * 120
$$

Where Sweep is the distance travelled by $\mathrm{S}$ relative to $\mathrm{O}$ to produce a pitch sweep from the lowest to the highest ex-tremes and displacement is the current distance from $\mathrm{S}$ to $\mathrm{O}$. The value of the Sweep can be set 
dynamically by the user and in effect represents the resolution of the auditory display. In Figure 3 the user chooses Sweep to be equal to $H$, the maximum range of the graph, and displacement to be the vertical displacement $h$.

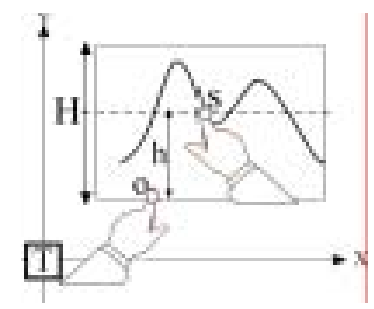

Figure 3: Vertical spacing of $O$ and $S$.

In normal exploratory mode, the pitch sequence produced at $\mathrm{S}$, when following the graph line with $\mathrm{S}$ and keeping $\mathrm{O}$ fixed on the bottom edge, represents the y-coordinates of the graph. In addition, we change the MIDI instrument de-pending on the sign of $h$. If $\mathrm{S}$ is above $\mathrm{O}$ then a piano in-strument renders the pitch. If $\mathrm{O}$ is above $\mathrm{S}$ then a xylo-phone is used. It is therefore easy to level $\mathrm{S}$ with $\mathrm{O}$ by monitoring the shift in instrument at that critical spatial relationship and so provides a simple way to compare sepa-rated points on the graph. Another important task is check-ing orientation of a tactile graph before use. This can be done in TouchMelody by keeping $\mathrm{O}$ fixed on the bottom edge of the diagram and $\mathrm{S}$ moved along the edge of the diagram. A stream of constant pitches informs the user that the diagram is horizontal.

We wanted to ensure our users had control over the sounds and could easily turn them on or off as needed. Our strategy allows $\mathrm{S}$ to produce sounds only if the elevation difference (spacing in the z-direction) between $\mathrm{O}$ and $\mathrm{S}$ is less than $1 \mathrm{~cm}$ i.e. both fingers in contact with the graph surface to provide control on the auditory output. If either of the fin-gers is lifted off the diagram the sounds stop.

\section{CONCLUSIONS AND FUTURE WORK}

Initial pilot testing with five blind-folded users show that the system can be understood easily, encourages the use of both hands for exploration and yields some unanticipated ways in which it can be used. Such uses include choosing different values of $H$ to control deliberately the shortest displacement to trigger a pitch change. For example, to study the changes a line graph undergoes in a particularly intricate region, some users chose $H$ to be small to magnify pitch changes corresponding to small changes in $h$. Indica-tions are that TouchMelody can facilitate or reinforce com-parative spatial judgments between two distant tactile cues. We will also provide synthesised speech cues so that the actual value of the displacement of $\mathrm{S}$ to $\mathrm{O}$ can be spoken to give an absolute value. We can use the FASTRACK to recognise simple gestures such as tapping to mark points of interest on the graph. We are also interested in investigating how this approach of augmenting tactile diagrams with spatial sound can impact their creation by the visually dis-abled users themselves. The technique described here pro-vides a way to enhance existing paper-based tactile diagrams with dynamic non-speech sounds, increasing their usefulness, usability and information content.

\section{REFERENCES}

1. American Printing House for the Blind. Guidelines for Design of Tactile Graphics. 2001. http://www.aph.org/ guides.htm

2. Challis, B. and Edwards, A.D.N. Design principles for tactile interaction. In Haptic Human-Computer Interac-tion (LNCS 2058), Brewster, S.A. and Murray-Smith, R. (Eds.), Springer, Berlin, Germany, 2001, 17-24.

3. Mansur, D.L., Blattner, M. and Joy, K. Sound-Graphs: A numerical data analysis method for the blind. Journal of Medical Systems 9 (1985), 163-174.

4. Ramloll, R., Yu, W., Riedel, B. and Brewster, S.A. Us-ing Non-speech Sounds to Improve Access to 2D Tabu-lar Numerical Information for Visually Impaired Users. In Proceedings of IHM-HCI 2001 (Lille, France) Springer, 2001, pp. 515-530. 
